\title{
DIVISION VI
}

\section{INTERSTELLAR MATTER}

\author{
MATIÈRE INTERSTELLAIRE
}

Division VI provides a focus for astronomers studying a wide range of problems related to the physical and chemical properties of interstellar matter in the Milky Way and other galaxies.

PRESIDENT
VICE-PRESIDENT
PAST PRESIDENT
BOARD

PRESIDENT VICE-PRESIDENT BOARD
Thomas J. Millar

You Hua Chu

John E. Dyson

Dieter Breitschwerdt, Michael G. Burton, Sylvie Cabrit, Paola Caselli, Elisabete M. de Gouveia Dal Pino, Gary J. Ferland, Mika J. Juvela, Bon-Chul Koo,S un Kwok, Susana Lizano, Michal Rozyczka, L. Viktor Tóth, Masato Tsuboi, Ji Yang

\section{DIVISION VI COMMISSIONS}

Commission $34 \quad$ Interstellar Matter

\section{DIVISION VI WORKING GROUPS}

\author{
Division VI WG \\ Division VI WG \\ Astrochemistry \\ Division VI WG \\ Star Formation \\ Planetary Nebulae
}

\section{TRIENNIAL REPORT 2006 - 2009}

\section{Introduction}

Division VI, which is itself a single Commission (Commission 34), has around 900 members whose research interests cover the wide spectrum of activities, theoretical, experimental and observational, associated with the study of the interstellar medium in the Universe. As such, the Division has close links with Divisions VIII, IX and X.

Our report on activity since 2006 is divided into four sections, covering important meetings and conferences, relevant proceedings published in the period under review, a list of important review articles, and Working Group reports.

\section{Meetings and conferences}

The reporting period has been one of great activity with many conferences devoted in whole or in part to the scientific interests of Division members. Below, we list some of the most significant meetings: 
- Planetary Nebulae in Our Galaxy and Beyond, IAU Symposium No. 234, 3-7 April 2006, HI, USA

- Triggered Star Formation in a Turbulent ISM, IAU Symposium No. 237, 14-18 August 2006, Prague, Czech Republic

- Science with ALMA, A New Era for Astrophysics, 13-17 November 2006, Madrid, Spain

- Molecular Databases for Herschel, ALMA, and SOFIA, 6-8 December 2006, Leiden, Netherlands

- 4th Korea-Mexico Joint Workshop on Astrophysics: Interstellar Medium, 5-7 May 2007, Daejeon, Korea

- Molecules in Space and Laboratory, 14-18 May 2007, Paris, France

- Asymmetrical Planetary Nebulae. IV, 18-22 June 2007, La Palma, Spain

- Star Formation Through Cosmic Time, 13-17 August 2007, Santa Barbara, CA, USA

- Massive Star Formation: Observations Confront Theory, 10-14 September 2007, Heidelberg, Germany

- From Protostellar Cores to Disk Galaxies, September 2007, Zurich, Switzerland

- FIR Workshop 2007, Far-Infrared and Submillimeter Emission of the Interstellar Medium: Models meet Extragalactic and Galactic Observations, 5-7 November 2007, Bad Honnef, Germany

- The Evolving Interstellar Medium in the Milky Way and Nearby Galaxies, 2-5 December 2007, Pasadena, CA, USA

- Magnetic Fields in the Universe. II. From Laboratory and Stars to the Primordial Structures, 28 January - 1 February 2008, Cozumel, Mexico

- Organic Matter in Space, IAU Symposium No. 251, 18-22 February 2008, Hong Kong, China

- The Local Bubble and Beyond. II, 21-24 April 2008, Philadelphia, PA, USA

- The Molecular Universe: an International Meeting on the Physics and Chemistry of the Interstellar Medium, 5-8 May 2008, Arcachon, France

- Protostellar Jets in Context, 7-12 July 2008, Rhodes, Greece

- 10th Asian-Pacific Regional IAU Meeting, 3-6 August 2008, Kunming, China

- The Role of Disk-Halo Interaction in Galaxy Evolution: Outflow vs. Infall?, 18-22 August 2008, Espinho, Portugal

- Praha2008, the 20th International Conference on High Resolution Molecular Spectroscopy, 2-6 September 2008, Prague, Czech Republic

- Cosmic Dust Near and Far, 8-12 September 2008, Heidelberg, Germany

- JENAM 2008, Symposium No. 7 on Grand Challenges in Computational Astrophysics, 8-11 September 2008, Vienna, Austria

- Cosmic Dust and Radiative Transfer, 15-17 September 2008, Heidelberg, Germany

- Future Directions in Ultraviolet Spectroscopy, 20-22 October 2008, Annapolis, MD, USA

- New Light on Young Stars: Spitzer's View of Circumstellar Disks, 26-30 October 2008, Pasadena, CA, USA

- Cosmic Magnetic Fields: from Planets, to Stars and Galaxies, 3-7 November 2008, Tenerife, Spain

- When the Universe Formed Stars, 17-21 November 2008, La Martinique Island, France

The Division is supporting a number of Symposia at the IAU XXVII General Assembly in Rio de Janeiro, August 2009, and is the coordinating Division for three Joint Discussions and two Special Sessions during the GA. 
- AKARI international conference, 16-22 February 2009, Tokyo, Japan (Exact title and the date are to be determined.)

- Gas and Dust Grains: From the ISM to the Solar System (Preliminary), 17-19 June 2009, Seoul, Korea

- Astrophysical Outflows and Associated Accretion Phenomena, IAU XXVII GA Joint Discussion (JD7), 6-7 August 2009, Rio de Janeiro, Brazil (Coordinating Division)

- FIR2009: the ISM of Galaxies in the Far-Infrared and Sub-Millimetre, IAU XXVII GA Joint Discussion (JD14), 12-14 August 2009, Rio de Janeiro, Brazil (Coordinating Division)

- Magnetic Fields in Diffuse Media, IAU XXVII GA Joint Discussion (JD15), 12-14 August 2009 (Coordinating Division)

- Young Stars, Brown Dwarfs, and Protoplanetary Disks, IAU XXVII GA Special Session (SpS7), 11-14 August 2009, Rio de Janeiro, Brazil (Coordinating Division)

- The Galactic Plane - In Depth and Across the Spectrum, IAU XXVII GA Special Session (SpS8), 11-14 August 2009, Rio de Janeiro, Brazil (Coordinating Division)

\section{Proceedings and monographs}

\subsection{Conference proceedings}

- Planetary Nebulae in our Galaxy and Beyond, Proc. IAU Symposium No. 234, Eds. M. J. Barlow \& R. H. Méndez (Cambridge: CUP), 2006.

- Far-Infrared and Submillimeter Emission of the Interstellar Medium, Eds. C. Kramer, S. Aalto \& R Simon, EAS-PS, 2007

- Triggered Star Formation in a Turbulent Interstellar Medium, Proc. IAU Symposium No. 237, Eds. B. G. Elmegreen \& J Palous (Cambridge: CUP), 2007

- Astrophysical Masers and Their Environments, Proc. IAU Symposium No. 242, Eds. J. M. Chapman \& W. A. Baan (Cambridge: CUP), 2008

- Massive Stars as Cosmic Engines, Proc. IAU Symposium No. 250, Eds. F, Bresolin, P. A. Crowther \& J. Puls (Cambridge: CUP), 2008

- Magnetic Fields in the Universe. II. From Laboratory and Stars to the Primordial Structures, Eds. A. Esquivel, et al., RMAA-CP, 2008, in press

- Asymmetrical Planetary Nebulae. IV, Eds. R. L. M. Corradi, A. Manchado \& N. Soker. See <www.iac.es/proyecto/apn4/pages/proceedings.php>.

\subsection{Research monographs}

- Physics and Chemistry of the Interstellar Medium, Sun Kwok, University Science Books, 2006

- The Elemental Abundances in Bare Planetary Nebula Central Stars and the Shell Burning in AGB Stars, Klaus Werner \& Falk Herwig, PASP, 118, 183, 2006

- Wonderful Mira, Christopher J. Wareing, 2008, Phil. Trans. R. Soc. A, 366. Triennial Issue Astronomy: Ed. J. M. T. Thompson,

\section{Review articles}

Recent articles on interstellar matter published in the Annual Reviews of Astronomy and Astrophysics have included:

- Snow, T. P., \& McCall, B. J. 2006, ARAEAA, 44, 367, Diffuse Atomic and Molecular Clouds

- Harris, D. E., \& Krawczynski, H. 2006, ARA\&A, 44, 463, X-Ray Emission from Extragalactic Jets 
- Bergin, E.A., \& Tafalla, M. 2007, ARA\&A, 45, 339, Cold Dark Clouds: The Initial Conditions for Star Formation

- Zinnecker, H., \& Yorke, H. W. 2007, ARA\&A, 45, 481, Toward Understanding Massive Star Formation

- McKee, C. F., \& Ostriker, E. C. 2007, ARA\&A, 45, 565, Theory of Star Formation

- Reynolds, S. P. 2008, ARA $\& A$, 46, 89, Supernova Remnants at High Energy

- Hester, J. J. 2008, ARA\&A, 46, 127, The Crab Nebula: An Astrophysical Chimera

- Tielens, A. G. G. M. 2008, ARAEA, 46, 339, Interstellar Polycyclic Aromatic Hydrocarbon Molecules

- Wyatt, M. C. 2008, ARA\&A, 46, 339, Evolution of Debris Disks

- Wang, L., \& Wheeler, J. C. 2008, ARA $\& A$, 46, 433, Spectropolarimetry of Supernovae

\section{Working Group reports}

\subsection{Working Group Astrochemistry}

Members: Ewine F. van Dishoeck (chair, Netherlands), Eric Herbst (secretary, USA), Louis J. Allamandola (USA), John H. Black (Sweden), Geoffrey A. Blake (USA), Paola Caselli (Italy, now UK), Pascale F. Ehrenfreund (Netherlands), Guido Garay (Chile), Micel Guelin (France), Christian Henkel (Germany), Uffe G. Jørgensen (Denmark), John P. Maier (Switzerland), Karl M. Menten (Germany), Thomas J. Millar (UK), Young Chol Minh (South Korea), Masatoshi Ohishi (Japan), Alejandro C. Raga (Mexico), Jonathan Rawlings (UK), Bertrand R. Rowe (France), and Ji Yang (China).

The Working Group on Astrochemistry planned and organized the highly successful symposium on Astrochemistry - Recent Successes and Current Challenges, IAU Symposium No. 231, held at Asilomar, California, USA, in August 2005. The volume of the proceedings, edited by Darek Lis, Geoffrey Blake \& Eric Herbst, which appeared in early 2006 , is a valuable addition to the field of astrochemistry.

Currently the Working Group is starting to prepare for the next symposium in the astrochemistry series, planned to be held in southern Europe in either 2010 or 2011. Chair Ewine van Dishoeck and secretary Eric Herbst have been stimulating other interim meetings in the field of astrochemistry and have also been searching for gifted young speakers for the next IAU Symposium, including related fields in chemical physics.

The astrochemistry working group website at <www.strw.leidenuniv.nl/ iau134> is regularly maintained and updated, especially on links with molecular databases.

Within the next several years, both the Herschel Space Observatory and the Atacama Large Millimeter Array (ALMA) will help to revolutionize the field of astrochemistry by dramatically improving the spatial resolution of small objects such as protoplanetary disks, vastly increasing the intensity of molecular spectral signals, and greatly enlarging the region of the electromagnetic spectrum available to study molecules. We trust that our next IAU Symposium will be bursting with new information and analysis.

\subsection{Working Group Star Formation}

Members: Francesco Palla (chair, Italy), Rafael Bachiller (Spain), Michael Burton (Australia), Lawrence E. Cram (Australia), Yasuo Fukui (Japan), Garay Garay (Chile), Thomas Henning (Germany), Charles J. Lada (USA), Maria T. V. T. Lago (Portugal), Susana Lizano (Mexico), Jan Palouš (Czech Republic), Bo Reipurth (USA), Annelia I. Sargent (USA), and Stephen E. Strom (USA). 
The Working Group on Star Formation has been involved in the organization of a number of IAU conferences on various aspects related to the field of star formation. In recent years, there has been a great interest and development both on the large scale aspects of star formation and feedback effects on the interstellar medium, and on the small scale implication on protoplanetary disk formation and evolution.

IAU Symposium No. 237 on Triggered Star Formation in a Turbulent Environment was held at the IAU XXVII General Assembly in Prague, August 2006. The proceedings of the conference edited by Bruce Elmegreen \& Jan Palous appeared in 2007 and represents a valuable reference book on the topic.

IAU Symposium No. 242 on Astrophysical Masers and Their Environments was organized in Alice Springs, Australia, in May 2007 and followed a previous IAU conference on masers held in Brazil in 2001. The volume of the Symposium was edited by J. M. Chapman \& W. A. Baan, appeared in 2008, and provides a thorough summary of the impact of maser research on star formation studies.

Finally, IAU Symposium No. 243 on Star-Disk Interaction in Young Stars was held in Grenoble in 2007. The proceedings have been edited by J. Bouvier \& I. Appenzeller and appeared in 2007.

The WG has been active at promoting and supporting requests for the organization of Symposia, Joint Discussions and Special Sessions during the IAU XXVII General Assembly in Rio de Janeiro, Brazil, 2009. As a result, we look forward to participating in IAU Symposium No.266 on Star Clusters - Basic Building Blocks throughout Time and Space, in JD7 on Astrophysical Outflows and Accretion Phenomena, and in SpS7 on Young Stars, Brown Dwarfs, and Protoplanetary Disks. The WG web page <www.arcetri.astro.it/sfwg is maintained and updated with links to forthcoming meetings on star formation.

\subsection{Working Group Planetary Nebulae}

Members: Arturo T. Manchado (chair, Spain), Michael J. Barlow (UK), Romano L. M. Corradi (Spain), You-Hua Chu (USA), Shuji Deguchi (Japan), Adam Frank (USA), George H. Jacoby (USA), Sun Kwok (Hong Kong, China), Alexander López (Mexico), Walter J. Maciel (Brazil), Roberto H. Méndez (USA), Quentin A. Parker (Australia), Detlef Schoenberner (Germany), Letizia Stanghellini (USA), and Albert Zijlstra (UK).

The Working Group on Planetary Nebulae planned and organized IAU Symposium No. 234 on Planetary nebulae in our Galaxy and Beyond, held in Waikoloa Beach (HI, USA), April 2006. The attendance was 150 astronomers from 18 countries. This meeting followed the time-honored tradition of having an IAU Symposium on planetary nebulae every five years. The symposium proceedings were edited by M. J. Barlow \& R. H. Mendez, and published timely in 2006. This volume offers contributions on a wide variety of subjects: Galactic and extragalactic PN surveys; the relationships between AGB stars, post-AGB objects, PN central stars and white dwarfs; nucleosynthesis and the properties of the central stars, including the role of binarity; PN properties across the full electromagnetic spectrum; atomic processes and PN abundances; mechanisms for the formation of PN structures; and properties and applications of extragalactic PNs, currently detected out to Coma cluster distances.

The WG is starting to plan its proposal for the next IAU Symposium in the planetary nebulae series, to take place on Tenerife, Spain, in summer 2011.

Some of the WG members have been organizing additional meetings in the field of planetary nebulae. Arturo Manchado (WG chair) \& Romano Corradi organized the conference Asymmetrical Planetary Nebulae. IV, held in June 2007 on La Palma, Spain. 
Albert Zijlstra, Romano Corradi \& Alberto Lopez, are organizing the follow-up conference Asymmetrical Planetary Nebulae. $V$, to be held in Bowness-on-Windermere, UK, in June 2010. See: <www. astrophysics.manchester.ac.uk/apn5.html>.

The WG web page <www.iac.es/proyecto/PNgroup/wg/index.html> is maintained and updated with links to past and forthcoming meetings on planetary nebulae.

Thomas J. Millar

president of the Division 